\title{
A Case Report of Calcifying Pseudoneoplasm of the Neuraxis: An Incidental Finding After a Car Accident
}

\author{
Farahnaz Bidari ZEREHPOOSH ${ }^{1}$, Mohammad SAMADIAN ${ }^{2}$, Vahideh Sadat AZHARI', Maryam BARANGI', \\ Kaveh EBRAHIMZADEH ${ }^{2}$, Mahnaz HEIDARY ${ }^{1}$
}

Department of 'Pathology and 'Neurosurgery, Loghman Hospital, TEHRAN, IRAN

\begin{abstract}
Calcifying pseudoneoplasms of the neuraxis are rare, poorly understood lesions that can occur anywhere in the central nervous system without any age or sex predilection. Their pathogenesis is still controversial. Patients can present with various symptoms due to lesion location and only few cases have been discovered incidentally. We present a 25 -year-old male referred to our hospital after a car accident. Computed tomography scan and magnetic resonance imaging was done because of the patient's head trauma and a calcified intracranial lesion was found and excised surgically. Pathologic evaluation of the specimen was consistent with the diagnosis of calcifying pseudoneoplasm of the neuraxis.
\end{abstract}

Key Words: Calcifying pseudoneoplasm of neuraxis, CAPNON

\section{INTRODUCTION}

Calcifying pseudoneoplasms of the neuraxis (CAPNONs), which are variably named as fibro-osseous lesions, cerebral calculi, brain stones or calcifying pseudotumors, are rare lesions that were first reported by Miller in 1922 (1); since then, 56 cases have been reported in international literature (1), none of them in the middle-eastern population. These lesions are typically benign, slow growing and non infiltrative, although their pathogenesis is controversial and various authors have considered them as metaplastic or reactive lesions $(1,2)$.

CAPNONs can occur anywhere within the neuraxis, intracranial or spinal, intra-axial or extra-axial (1-3). Although typically benign, symptoms of CAPNONs typically present secondary to compression and surrounding mass effect (4). Various symptoms and signs will therefore appear according to their location. Intracranial ones often present with headache, seizure and/or other symptoms related to the local compressive effect of the mass $(1,2)$; on the other hand spinal lesions commonly present with localized pain in the affected area (1). Only 3 out of 56 reported patients were incidentally found (8.1\%) (1). In this report we describe an intra-axial lesion in the temporal lobe which was an incidental finding during diagnostic procedures in a car accident case.

\section{CASE REPORT}

A 25-year-old man with a healthy past history and no previous neurological problem suffered a car accident and underwent a computed tomography (CT) scan which was followed by a magnetic resonance imaging (MRI) scan. CT scan revealed a $3 \mathrm{~cm}$ calcified ovaloid mass in the left medial temporal region that was suspected to be a meningioma (Figure 1). T1 and T2W MRI showed a hypointense mass in the left medial temporal lobe with heterogenous enhancement of the lesion (Figure 2-4). The patient elected to undergo mass resection surgery. After general anesthesia in the left lateral decubitus position, left temporal craniotomy was performed. The surgical approach was through an inferior temporal gyrus corridor, and after microsurgical dissection of the inferior temporal gyrus the calcified mass was totally resected. The postoperative period was uneventful and the patient is now well after 3 years.

Microscopic examination revealed amorphous calcification and psammoma bodies with foci of ossification in the brain tissue (Figure 5) and a loose fibromyxoid stroma in some parts that contained scattered blood vessels (Figure 6). Calcified areas were sometimes surrounded by cells resembling meningothelial cells and also palisaded spindle cells and few multinucleated giant cells. No epithelial component was seen. 


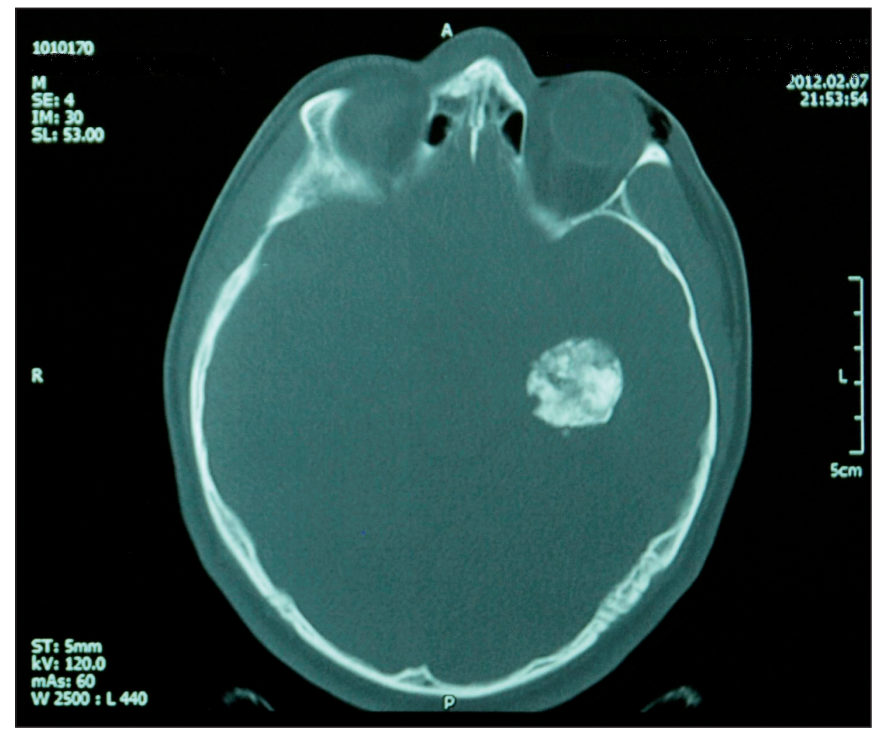

Figure 1: Axial bone window CT scan showed a calcifying mass in left medial temporal region.

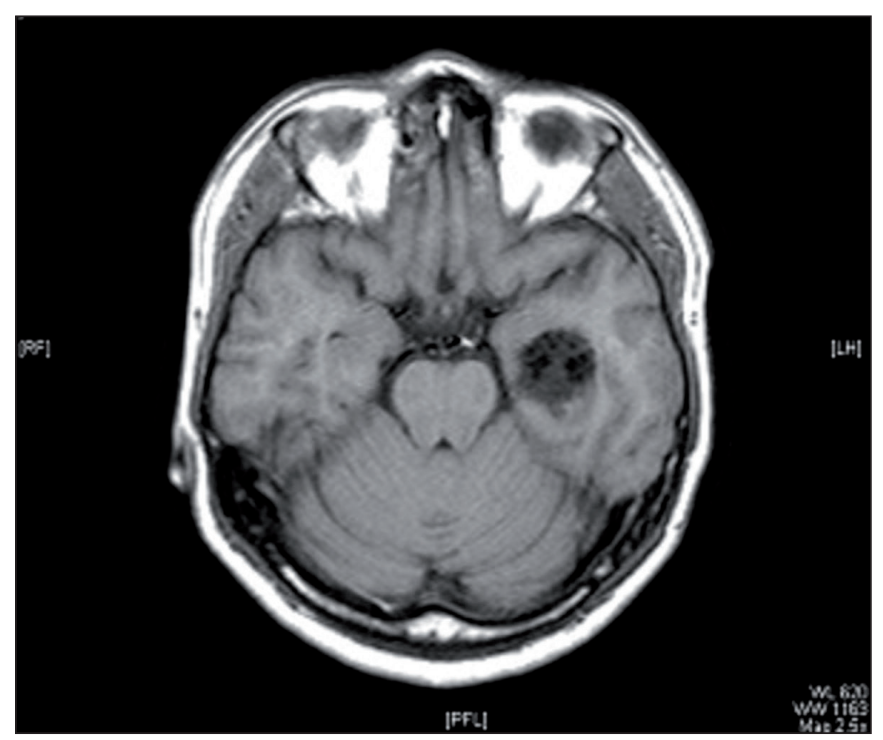

Figure 3: Axial T1W MRI showed a hypointense mass in the left medial temporal lobe.

Immunohistochemical staining was performed using commercially (DAKO), available antibodies against EMA, NSE, S-100, vimentin, CD68 and glial fibrillary acidic protein (GFAP). Tumor cells were negative for epithelial membrane antigen (EMA), neuron specific enolase (NSE), S-100 protein and GFAP. Few vimentin and CD68 positive cells are seen scattered in the surrounding calcified areas.

The final diagnosis was calcifying pseudoneoplasm of the neuraxis.

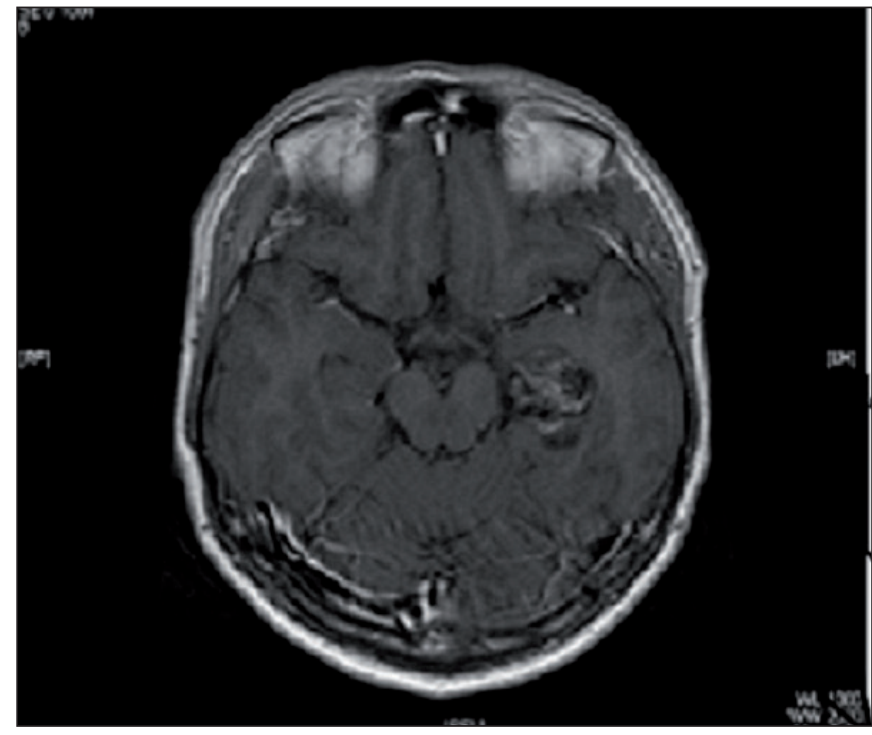

Figure 2: Axial T1W MRI with Gd revealed heterogenous enhancement of the lesion.

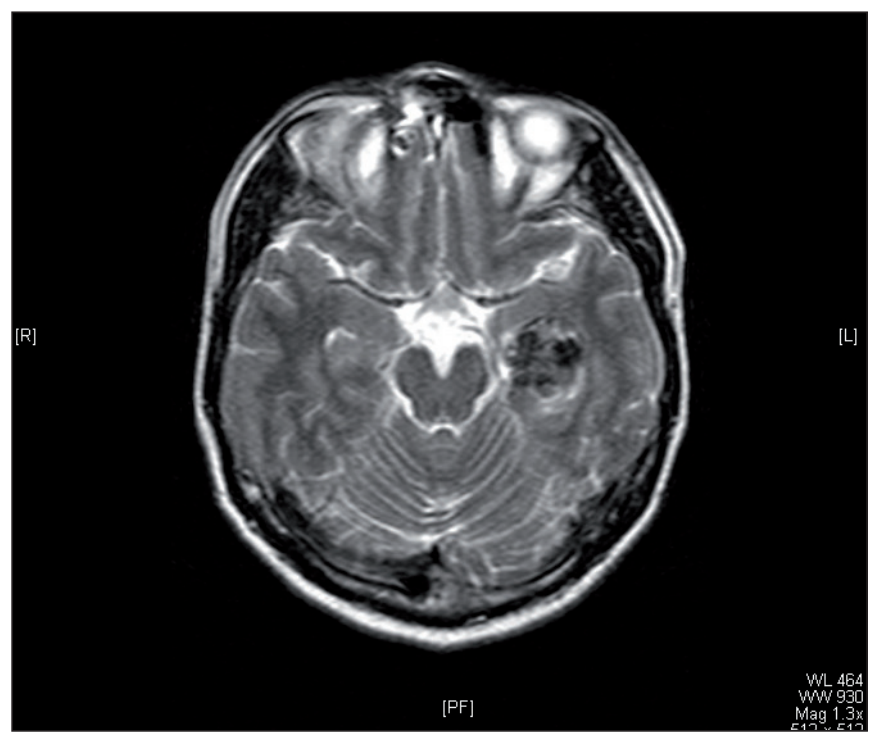

Figure 4: Axial T2W MRI showed a hypointense mass in the left temporal lobe.

\section{DISCUSSION}

CAPNONs are rare lesions arising anywhere in the central nervous system (CNS) (1-3,5). These lesions are intraaxial or extraaxial (1), and mostly dural-based (7); intracranial ones often involve the leptomeninges whereas spinal cases generally involve paraspinous meninges, soft tissue and bone (8). CAPNONs have been mentioned in the literature since 1922 as unusual fibro-osseous lesions of the brain $(1,6)$. They were recognized as a distinct entity in 1978 and there are 40 international reported cases $(1,7,9)$. 


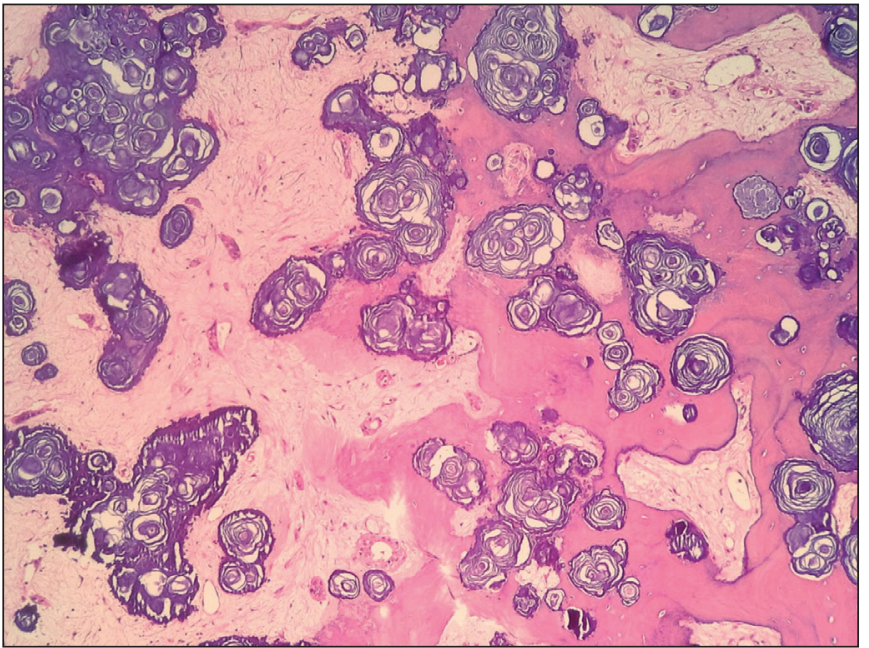

Figure 5: Psammomatous calcification with foci of ossification in brain tissue and no epithelial component (H\&E; x100).

Most of the published articles mention no sex or age predominance with an age range of 6-83 years $(1,3)$. Headache and seizure are the most common symptoms in intracranial lesions and only few cases are discovered incidentally $(1,2)$. The typical lesion is sporadic, but some CNS lesions have occurred in association with meningioangiomatosis in patients with neurofibromatosis type 2 (8).

The original tissue is controversial; a meningothelial origin is suspected in dural-base lesions while the hard dense fibrous stroma and the spindle cell population make a mesenchymal origin another possibility $(3,7)$.

The pathogenesis of CAPNON is not well understood but based on the available data a reactive process is more possible than neoplastic ones (1). It has been suggested, but not proven, that CAPNON may develop as a response to multiple inciting factors, which can account for the variations in histopathologic features. The causal factors are not yet understood, but response to possible trauma, infection, or inflammation has been proposed (3).

In a few cases, CAPNONs have been reported in combination with neoplasms such as ganglioglioma or dysembryoplastic neuroepithelial tumor $(2,10)$

On imaging, they can easily be confused with malignant lesions such as chondrosarcoma or chondroblastoma or even more benign pathologies such as meningioma (4). CT images typically show solid areas of calcification and MR imaging often shows a well-defined lesion that is hypointense in $\mathrm{T} 1$ and $\mathrm{T} 2$-weighted images without any

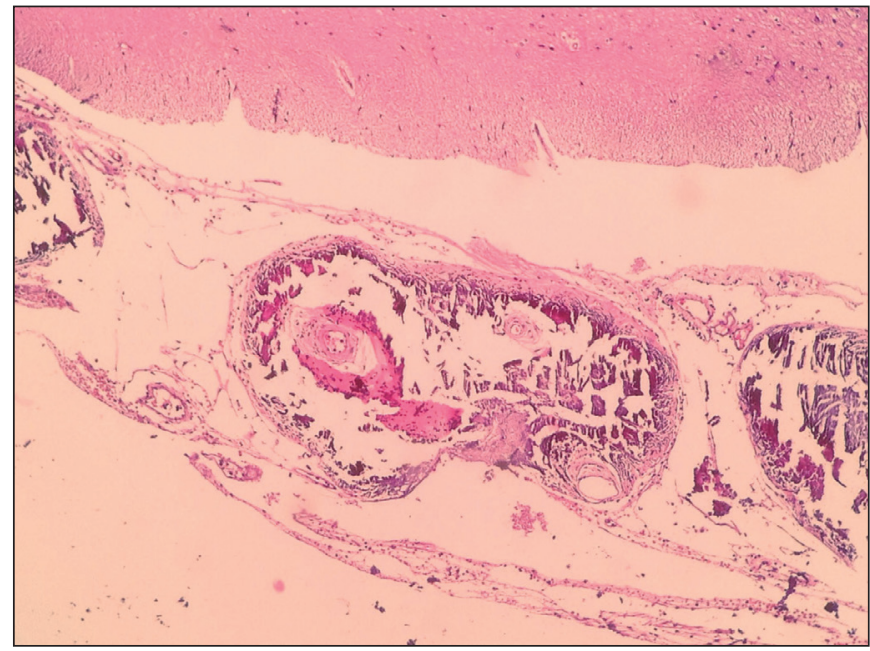

Figure 6: The fibromyxoid stroma contained scattered blood vessels and areas of amorphous granular calcification surrounded by spindle cells (H\&E; $\mathrm{x} 100)$.

brain edema. Contrast enhancement is limited to minimal linear internal or rim enhancement $(1,11)$.

The above CT and MRI findings were also seen in this presented case but with heterogenous contrast enhancement. Surprisingly similar CT and MRI findings to those of our case were seen in the case reported by Pereira et al. as a choroid plexus papilloma with osseous metaplasia and this similarity should be kept in mind in the differential diagnosis of such imaging presentations (11).

The classic histopathological features of CAPNON include a set of common elements: 1) presence of chondromyxoid matrix in a nodular pattern; 2) palisading spindle to epithelioid cells; 3) fibroblastic proliferation; 4) amorphous calcification, psammoma bodies and osseous metaplasia; 5) foreign-body reaction with giant cells surrounding the calcified region $(1,3,7)$. These pathological presentations are not evident in each case and some lesions may not show all of the above features (3). Our presented case revealed all of the abovementioned histopathological features except for the nodular chondromyxoid matrix.

The microscopic differential diagnosis of CAPNON includes a variety of reactive lesions such as granulomatous diseases, herniated disc material, and some neoplastic lesions such as chondroid chordoma, chondroma, chondrosarcoma, metaplastic meningioma, choroid plexus papilloma with osseous metaplasia and calcified primary parenchymal central nervous system tumors $(1,11)$.

A wide variety of immunohistochemical stains have been used to characterize CAPNONs. The majority of recent 
reports have focused on S100 protein, vimentin, EMA, and GFAP $(1,2)$. All CAPNONs stained positive for vimentin, most stained positive for EMA, and were negative for $\mathrm{S} 100$ and GFAP. A minority of cases stained positive for S100, were negative for EMA, and 1 case stained positive for GFAP (2).

The distinctive histopathology of CAPNON makes it easy to diagnose for expert pathologists. To the unwary, however, it can be mistaken for other tumors with extensive calcification, such as psammomatous meningioma. Calcifications in meningioma are smaller, round, and lamellated in contrast to the large, irregular calcifications of CAPNON. Choroid plexus papilloma with osseous metaplasia can be differentiated from CAPNON with the presence of epithelial components (11). Spinal lesions may mimic calcified tuberculosis, but CAPNON does not form granulomas, despite nodules of peripheral cellularity and occasional giant cells (8).

\section{REFERENCES}

1. Nonaka Y, Aliabadi HR, Friedman AH, Odere FG, Fukushima T. Calcifying pseudoneoplasms of the skull base presenting with cranial neuropathies: Case report and literature review. J Neurol Surg Rep. 2012;73:41-47.

2. Kerr EE, Broys E, Bobinski M, Shahlaie K. Posterior fossa calcifying pseudoneoplasm of the central nervous system. J Neurosurg. 2013;118:896-902.
3. Aiken AH, Akgun $\mathrm{H}$, Tihan T, Barbaro N, Glastonbury C. Calcifying pseudoneoplasms of the neuraxis: CT, MR imaging, and histologic features. Am J Neuroradiol. 2009;30:1256-60.

4. Alshareef M, Vargas J, Welsh C, Kalhorn SP. Calcifying pseudoneoplasm of the cervicomedullary junction: Case report and a literature review. World Neurosurg. 2016;85:364.e11-8.

5. Lyapichev K, Bregy A, Shah AH, Shah K, Desai MB, Petito C, Komotar RJ. Occipital calcified pseudoneoplasms of the neuraxis (CAPNON): Understanding a rare pathology. BMJ Case Rep. 2014;2014. pii: bcr2014206855.

6. Miller EA. Calculi within the brain. Report of a case of intracranial calcification with successful operation and recovery. Surg Gynecol Obstet 1922;34:786-9.

7. Mohapatra I, Manish R, Mahadevan A, Pasad C, Sampath S, Shankar SK. Calcifying pseudoneoplasm (fibro osseous lesion) of neuraxis (CAPNON) - a case report. Clin Neuropathol. 2010;29:223-6.

8. Donev K, Scheithauer BW. Pseudoneoplasms of the nervous system. Arch Pathol Lab Med. 2010;134:404-16.

9. Bartanusz V, Ziu M, Jimenezand DF, Henry JM. Calcifying pseudoneoplasm of the atlantoaxial joint in a child. J Neurosurg Spine. 2013;18:367-71.

10. Yan X, Marsh WR, Scheithauer BW. Dysembryoplastic neuroepithelial tumor and calcifying pseudoneoplasms of the neuraxis: A collision of two seizure-associated lesions. Clin Neuropathol. 2011;30:197-202.

11. Pereira DB, Gasparetto EL, Marcondes de Souza J, Chimelli L. Choroid plexus papilloma with osseous metaplasia as a differential diagnosis of calcifying pseudoneoplasms of the neuraxis. Am J Neuroradiol. 2010;31:E51-2. 